\title{
A symptomatic bladder rhabdomyosarcoma managed by a partial cystectomy with bilateral ureterostomy for a 2-year-old male: A
}

\author{
rare case report
}

Marah Mansour ${ }^{1}$, Manar Ibrahim ${ }^{1}$, Aamer Hammod ${ }^{1}$, Tamim Alsuliman ${ }^{2}$, and Mayas

Yousif $^{3}$

${ }^{1}$ Faculty of Medicine, Tartous University

${ }^{2}$ Saint-Antoine Hospital, AP-HP, Sorbonne University

${ }^{3}$ Imam Zayn al-Abidin Hospital

October 4, 2021

\begin{abstract}
Bladder Rhabdomyosarcoma is a rare disease. It can be diagnosed by imaging and histological examination and managed using multi-modal therapy. Herein, we report a large non-metastatic rhabdomyosarcoma mass positioned in the bladder which was successfully eradicated through open surgery.
\end{abstract}

A symptomatic bladder rhabdomyosarcoma managed by a partial cystectomy with bilateral ureterostomy for a 2-year-old male: A rare case report

A symptomatic bladder rhabdomyosarcoma managed by a partial cystectomy with bilateral ureterostomy for a 2-year-old male: A rare case report

\begin{abstract}
Bladder Rhabdomyosarcoma is a rare disease. It can be diagnosed by imaging and histological examination and managed using multi-modal therapy. Herein, we report a large non-metastatic rhabdomyosarcoma mass positioned in the bladder, which was successfully eradicated through open surgery.
\end{abstract}

\section{Clinical Key Message}

Bladder rhabdomyosarcoma should be considered in case of soft tissue masses with gross hematuria. However, early diagnosis and treatment are recommended in order to improve the quality of life and the survival rate of the patients.

Keywords: Rhabdomyosarcoma; bladder; hematuria; partial cystectomy; metastasis; abdominal; chemotherapy; cystectomy; bilateral ureterostomy

A symptomatic bladder rhabdomyosarcoma managed by a partial cystectomy with bilateral ureterostomy for a 2-year-old male: A rare case report

\section{Introduction}

Rhabdomyosarcoma (RMS) is a rare malignant tumor of soft tissue, most commonly detected in children and young adults. It makes up about 3-4\% of childhood cancer (Kim JR, 2017 Aug; Panda et al., 2017 May). RMS can arise anywhere in the body where there are muscles; in the extremities, head and neck, and the genitourinary tract (Mandeville, $2019 \mathrm{Jul}$ ). Imaging is an important diagnostic procedure, whereas; 
computerized tomography (CT) scans of primary sites with contrast or magnetic resonance imaging (MRI) are considered one of the most reliable diagnostic investigations (Panda et al., 2017 May). The differential diagnosis (DDx) of pediatric RMS includes Lipomas, Liposarcoma, Lymphadenopathy, Lymphoproliferative disorders, Neurofibromatosis type I, Osteosarcoma, Wilms tumor, and Ewing's sarcoma (Matondang et al., 2020 Apr). The Prognosis for RMS has become more promising because of multi-modal therapy including surgery, chemotherapy, and radiotherapy (Panda et al., 2017 May). Herein, we report a non-metastatic stage 3 bladder RMS in a two-year-old male.

\section{Case Presentation}

A two-year-old male was admitted to the Department of Pediatric Surgery complaining of gross hematuria and urinary frequency for the last two weeks. Apart from the above-mentioned medical history, clinical examination demonstrated a lower abdominal soft mass. Urinalysis results have revealed regular erythrocyte cells. Digital rectal exam (DRE) was negative. An abdominal ultrasound (US) had demonstrated a pelvic mass compressed in the posterior bladder wall (Fig.1). A CT scan with a bladder contrast demonstrated a pelvic mass, measuring $\sim 6 \times 7 \mathrm{~cm}$, located in the bladder with no distant metastasis and no lymph node enlargement (Fig.2). Those findings strengthened the suspicion of RMS/Wilms tumors as DDx. A transurethral endoscopy for diagnostic purposes with the possibility of a total cystectomy was performed (Fig.3). A large, inclusive, and prominent mass in the bladder was found. A biopsy was ordered to confirm the final diagnosis. Due to the lack of access to the chemotherapy centers and the worsened symptoms of the patient, the patient family's desired to perform a palliative treatment. Therefore, a complete excisional biopsy was done, referring to the clear surgical margins and the absence of distant metastasis. A partial cystectomy of the bladder with the preservation of the bladder triangle and the conduction of bilateral ureterostomy was successfully performed (Fig.4) before the conduction of chemotherapy. Stents were also bilaterally inserted in the ureters using a catheter in order to prevent potential stenosis. Histopathological study revealed a dense cambium layer of primitive malignant cells under an intact epithelial surface and a gross appearance of multiple polypoid nodules with hypocellular areas containing a myxoid stroma (Fig.5). Tumor cells had invaded the bladder muscularis propria (Detrusor muscle). Immunohistochemical studies showed positive nuclear expression of Myogenin and Actin where both of them were expressed in large cells showing morphological evidence of skeletal muscle differentiation, assuring that the final diagnosis was Multifocal embryonal RMS. The child's condition was good with no significant complaints. He was dismissed and referred to the Urology and chemotherapy centers; however, the patient died five months post-operation and before the conduction of chemotherapy. The etiology is not fully known, it is suggested to be a result of delayed diagnosis and conduction of chemotherapy, advanced tumor stage, invasion of the bladder muscles, and patient age.

\section{Discussion and Conclusion}

RMS is the most common soft-tissue sarcoma of childhood and comprises $3.5 \%$ of childhood cancers seen in the 0-14 y age group (Panda et al., 2017 May). RMS can affect any part of the body; however, the most common primary sites are the head and neck region, $36 \%$, genitourinary tract $23 \%$, extremities $19 \%$, and others 22\% (Panda et al., 2017 May). RMS is histologically classified into three subtypes; embryonal, alveolar, and polymorphic type. The most common subtype is embryonic, which makes up $70-80 \%$ of cases (Panda et al., 2017 May). We report a large embryonal RMS of the bladder in a two-year-old male, which was managed by partial surgical excision of the bladder with bilateral ureterostomy (Fig.4). Rhabdomyosarcoma of the bladder/prostate symptoms are gross hematuria, difficulty in voiding, urinary retention, or urgency (Wu, 2013 Mar). Our patient only presented with gross hematuria and urinary frequency; routinely found in both Wilms tumor and RMS. The initial diagnosis for patients with suspected rhabdomyosarcoma should include a CT scan or MRI scan of the tumor (Kim JR, 2017 Aug). In our case, a CT with a bladder contrast scan demonstrated a rounded; measuring $\sim 6 \mathrm{x} 7 \mathrm{~cm}$ in diameter, situated in the trigone region of the bladder adhered to the posterior wall of the bladder, which was projected towards the lumen with no distant metastasis (Fig.2). Since neither area of calcification or fat density regions, thrombus in the renal veins nor involvement of regional lymph nodes were found; indicating more to findings of Wilms 
tumor on a CT scan (Astuti et al., 2012 Feb 5), RMS was highly suspected. Prior studies have found that $27.5 \%$ of distant metastatic cases were significantly associated with unfavorable primary tumor site and tumor size (Kim JR, $2017 \mathrm{Aug}$ ); however, our case included an unfavorable site and a tumor larger than $5 \mathrm{~cm}$ but no distant metastases (Fig.5). A transurethral endoscopy and an open incisional biopsy were performed (Fig.3); which were the most appropriate procedures in our case since the patient was young, and his tumor was large (Schalow \& Broecker, $2003 \mathrm{Jul}$ ). In contrary to a previous study that has proven that completeness of tumor resection risk of failure increased by $63 \%$ for small children with large tumors (Rodeberg et al., 2011 Jun 1), the pathological study in our case indicated tumor-free surgical margins and no signs of vascular/lymphatic invasion. According to the CT scan images, pathological study, and intergroup rhabdomyosarcoma study group, the tumor was at stage 3 (Fig.2, Fig.5) (Panda et al., 2017 May). Immunohistochemical studies of RMS showed positive nuclear staining with antibodies to MyoD1 and/or Myogenin, compared to non-RMS pediatric tumors (Sebire \& Malone, 2003 Jun). In cases with differentiated tumor cells, the positive nuclear expression of Myogenin was stronger than MyoD1 (Sebire \& Malone, 2003 Jun). Considering this, nuclear Myogenin and Actin staining were used in our patient, where specimens demonstrated the positive nuclear expression of Myogenin and Actin with skeletal muscle differentiation, leading to the definite diagnosis of RMS. The latest recommendation on the management of RMS involves surgery, chemotherapy, and radiotherapy (Schalow \& Broecker, $2003 \mathrm{Jul}$ ). In contrast to the literature, our patient subsequently underwent an open surgery using a Pfannenstiel incision, where a partial cystectomy of the bladder with the preservation of the bladder triangle and the conduction of bilateral ureterostomy was performed (Fig.4). Stents were also bilaterally inserted in the ureters using a catheter in order to prevent potential stenosis. Due to therapeutic challenges (lack of access to the chemotherapy centers, the patient's family decision, and the worsened symptoms of the patient), the chemotherapy could not be conducted. As a palliative treatment, the surgery was the only treatment of choice for this patient, considering the clear margins and no distant metastasis. In this case, an excisional biopsy was done and confirmed the final diagnosis of multifocal embryonal RMS (Fig.5). The patient's condition was good with no major complaints. Although the surgery was successful with no significant complications, the patient died five months later. The etiology is not clearly known, it is suggested to be a result of delayed diagnosis and conduction of chemotherapy, advanced tumor stage, invasion of the bladder muscles, and patient age. In conclusion, whilst the RMS of the bladder is rare in children, it should be considered as a DDx in case of soft tissue masses with gross hematuria. Early diagnosis and treatment could improve the quality of life and the survival rate of the patients. The management could be more difficult in the case of fragile health systems.

\section{Author's contributions}

M.M: design of study, data collection, data interpretation and analysis, drafting, critical revision, approval of final manuscript.

M.I: data collection, data interpretation and analysis, critical revision, drafting, approval of final manuscript.

A.H: data interpretation and analysis, critical revision, drafting, approval of final manuscript.

T.A: drafting, critical revision, approval of final manuscript.

M.Y: The Supervisor, patient care, drafting, critical revision, approval of final manuscript

\section{References}

1. Astuti, D, Mark R Morris, Wendy N Cooper, Raymond H J Staals, Naomi C Wake, Graham A Fews , . . Maher, E. R. (2012 Feb 5). Germline mutations in DIS3L2 cause the Perlman syndrome of overgrowth and Wilms tumor susceptibility. Nature genetics. , 44 , 277-284https://doi.org/10.1038/ng.1071

2. Kim JR, Y. H., Koh KN, Jung AY, Cho YA, Lee JS. (2017 Aug). Rhabdomyosarcoma in children and adolescents: patterns and risk factors of distant metastasis. American Journal of Roentgenology , 209 , 409-416.https://doi.org/10.2214/AJR.16.17466.

3. Mandeville, H. C. (2019 Jul). Radiotherapy in the management of childhood rhabdomyosarcoma. Clinical Oncology , 31 , 462-470.https://doi.org/10.1016/j.clon.2019.03.047 
4. Matondang, S., Suwita, B. M., Budianto, T., \& Dwina, Y. (2020 Apr ). Rhabdomyosarcoma of the prostate with bladder and urethral infiltration: A case report. Urology case reports ,10;31:101199.https://doi.org/10.1016/j.eucr.2020.101199.

5. Panda, S. P., Chinnaswamy, G., Vora, T., Prasad, M., Bansal, D., Kapoor, G., . . Bakhshi, S. (2017 May). Diagnosis and management of rhabdomyosarcoma in children and adolescents: ICMR consensus document. The Indian Journal of Pediatrics. , 84, 393-402.https://doi.org/10.1007/s12098-017-2315-3

6. Rodeberg, D. A., Julie A Stoner, Norbert Garcia-Henriquez, R Lor Randall , \& Sheri L Spunt, C. A. A., Simon Kao, Charles N Paidas, Lynn Million, Douglas S Hawkins. (2011 Jun 1). Tumor volume and patient weight as predictors of outcome in children with intermediate risk rhabdomyosarcoma: a report from the Children's Oncology Group.cancer, 117, 2541-2550.https://doi.org/10.1002/cncr.25719

7. Schalow, E. L., \& Broecker, B. H. (2003 Jul). Role of surgery in children with rhabdomyosarcoma. Medical and pediatric oncology. , 41, 1-6.https://doi.org/10.1002/mpo.10261

8. Sebire, N. J., \& Malone, M. (2003 Jun). Myogenin and MyoD1 expression in paediatric rhabdomyosarcomas. Journal of clinical pathology, 56, 412-416.https://doi.org/10.1136/jcp.56.6.412

9. Wu, H.-Y. (2013 Mar). The surgical management of paediatric bladder and prostate rhabdomyosarcoma. Arab journal of urology ,11 , 40-46.https://doi.org/10.1016/j.aju.2012.11.003

\section{Figures Explanation}

Fig.1: An abdominal ultrasound demonstrated a pelvic mass compressed in the posterior bladder wall.

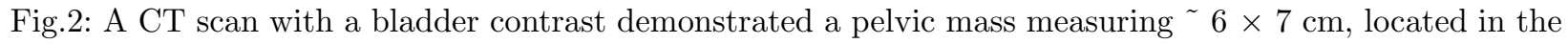
bladder, with no distant metastasis and no lymph node enlargement.

Fig.3: A transurethral endoscopy revealed a large, inclusive, and prominent mass in the bladder.

Fig.4: The abdominal mass was eradicated by a partial cystectomy of the bladder with bilateral ureterostomy.

Fig.5: The specimen revealed a dense cambium layer of primitive malignant cells under an intact epithelial surface and a gross appearance of multiple polypoid nodules with hypocellular areas containing a myxoid stroma.

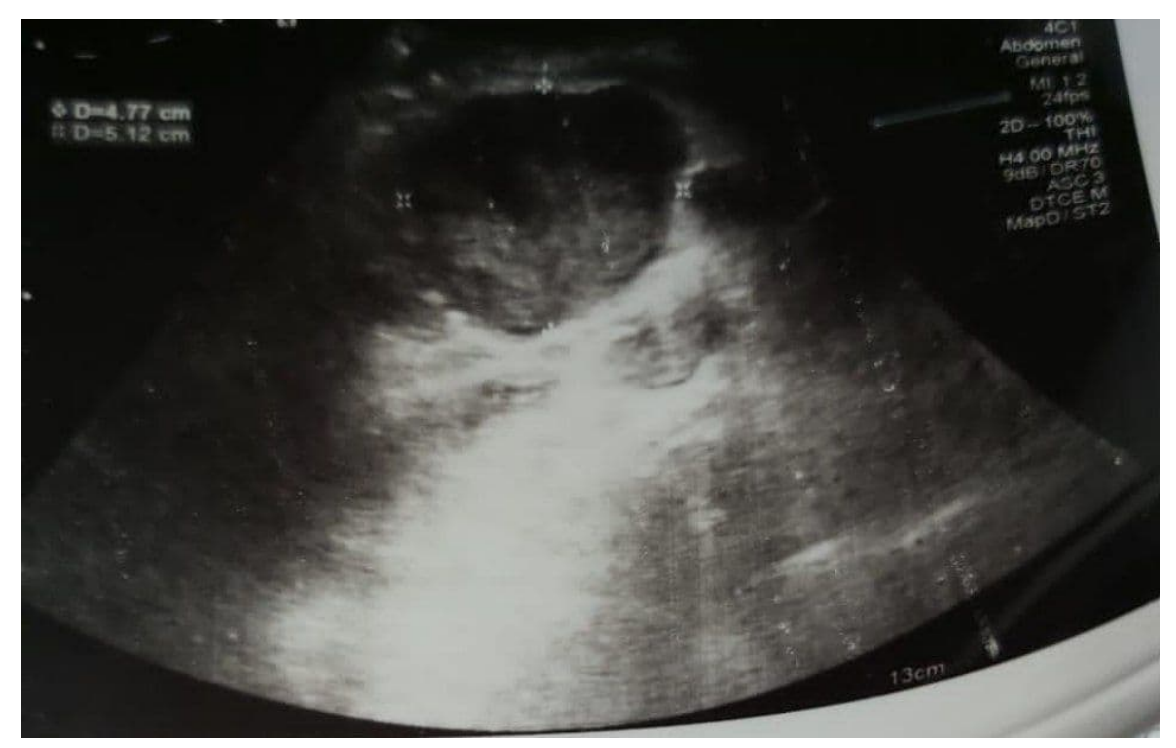




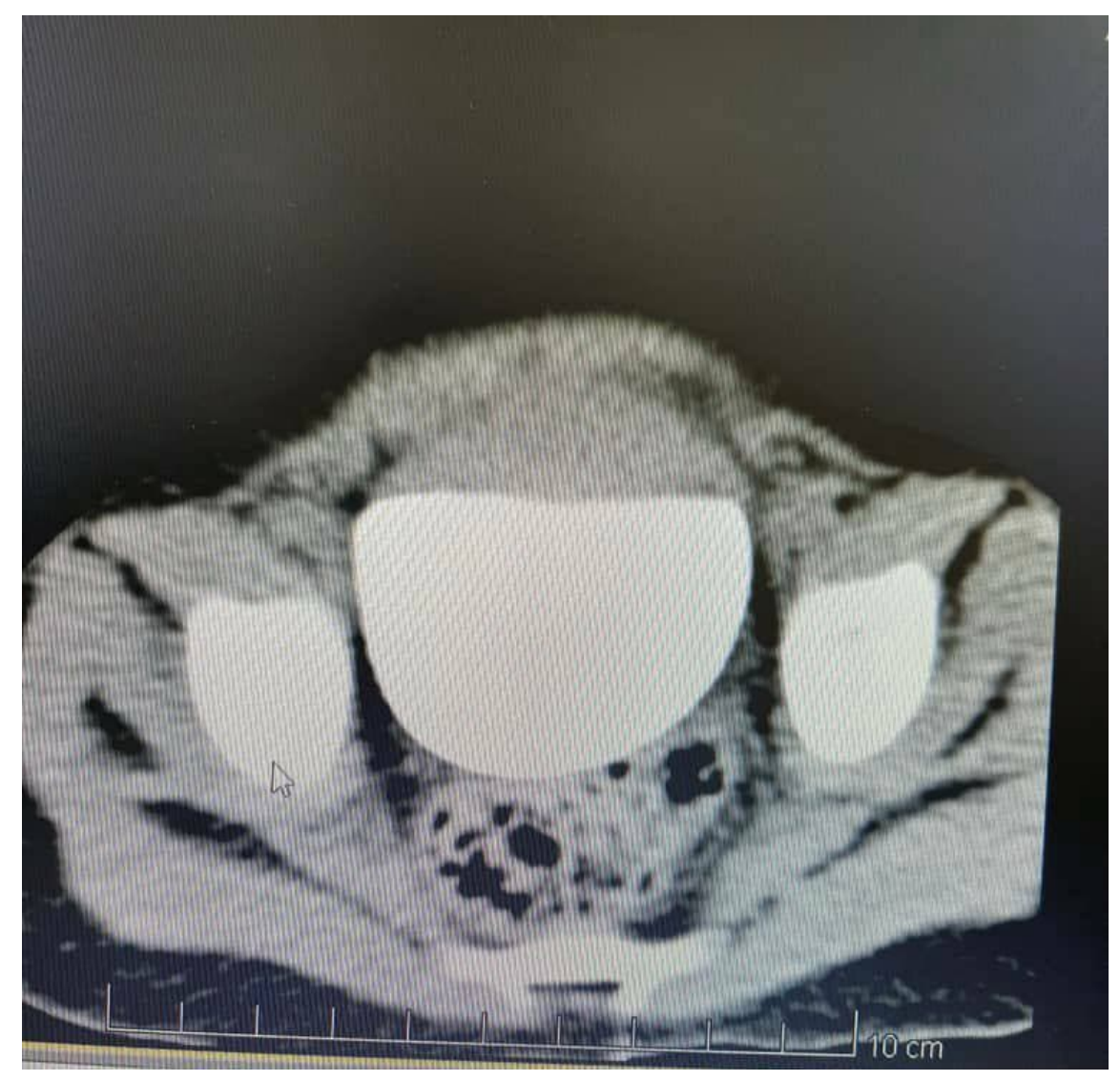




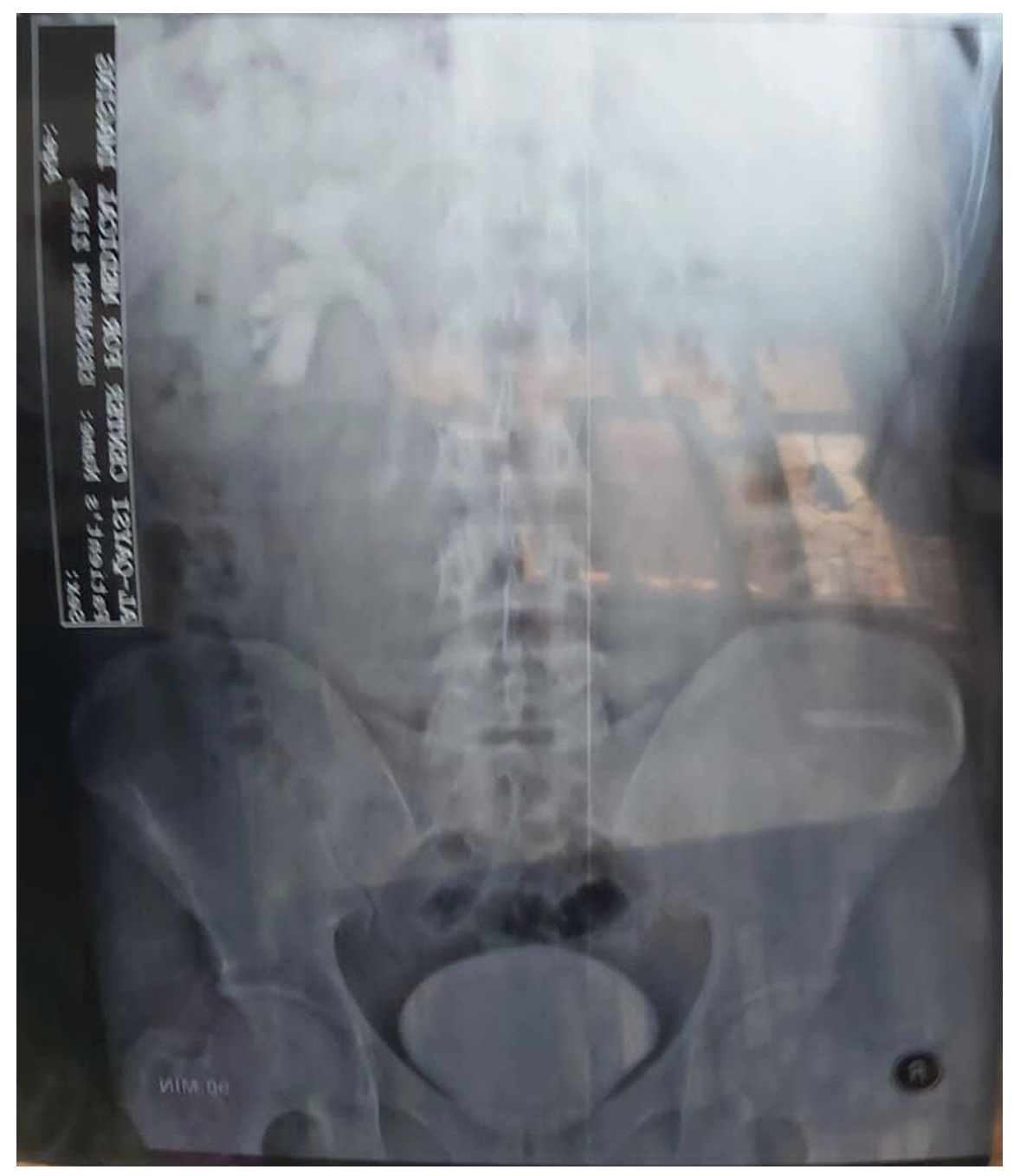




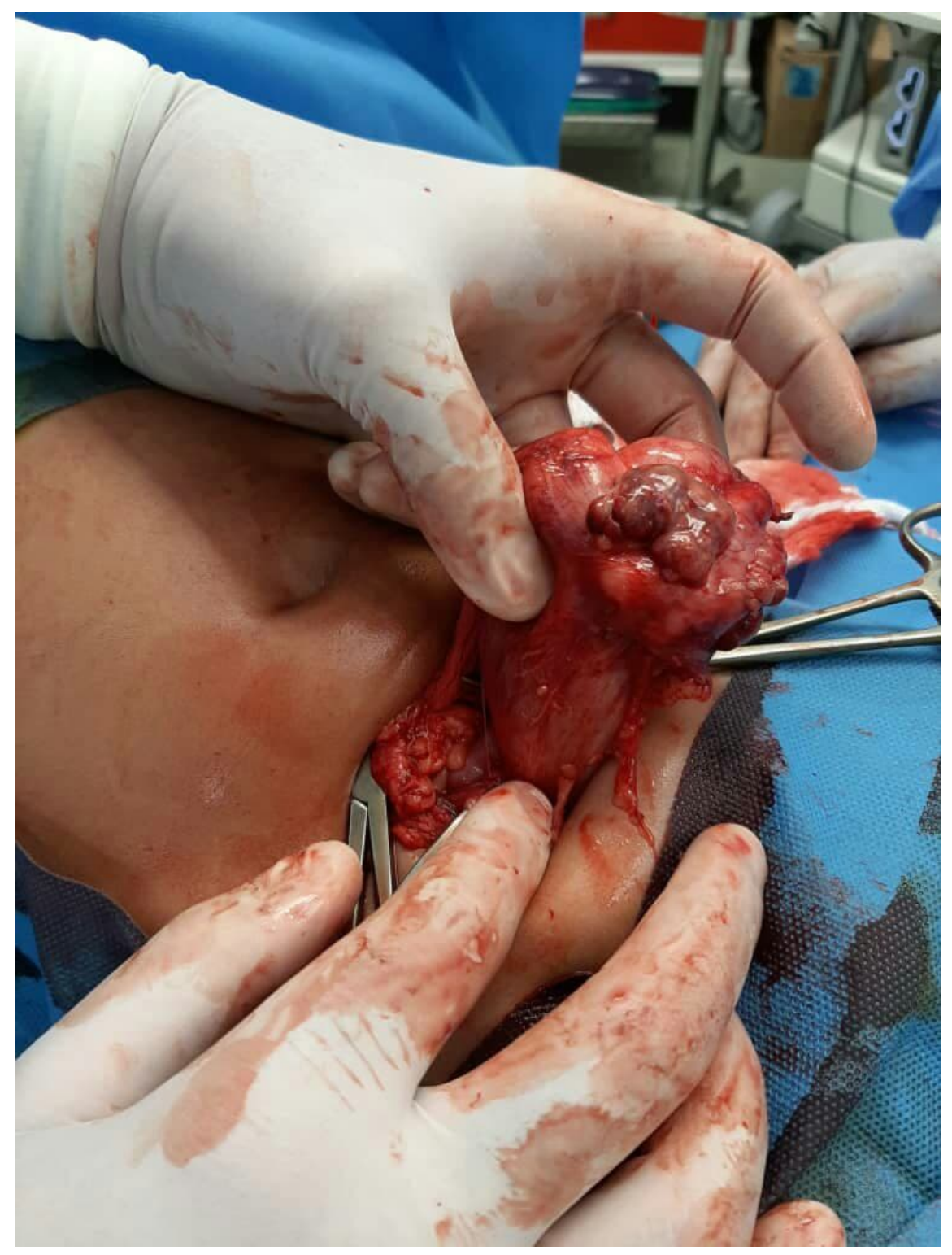




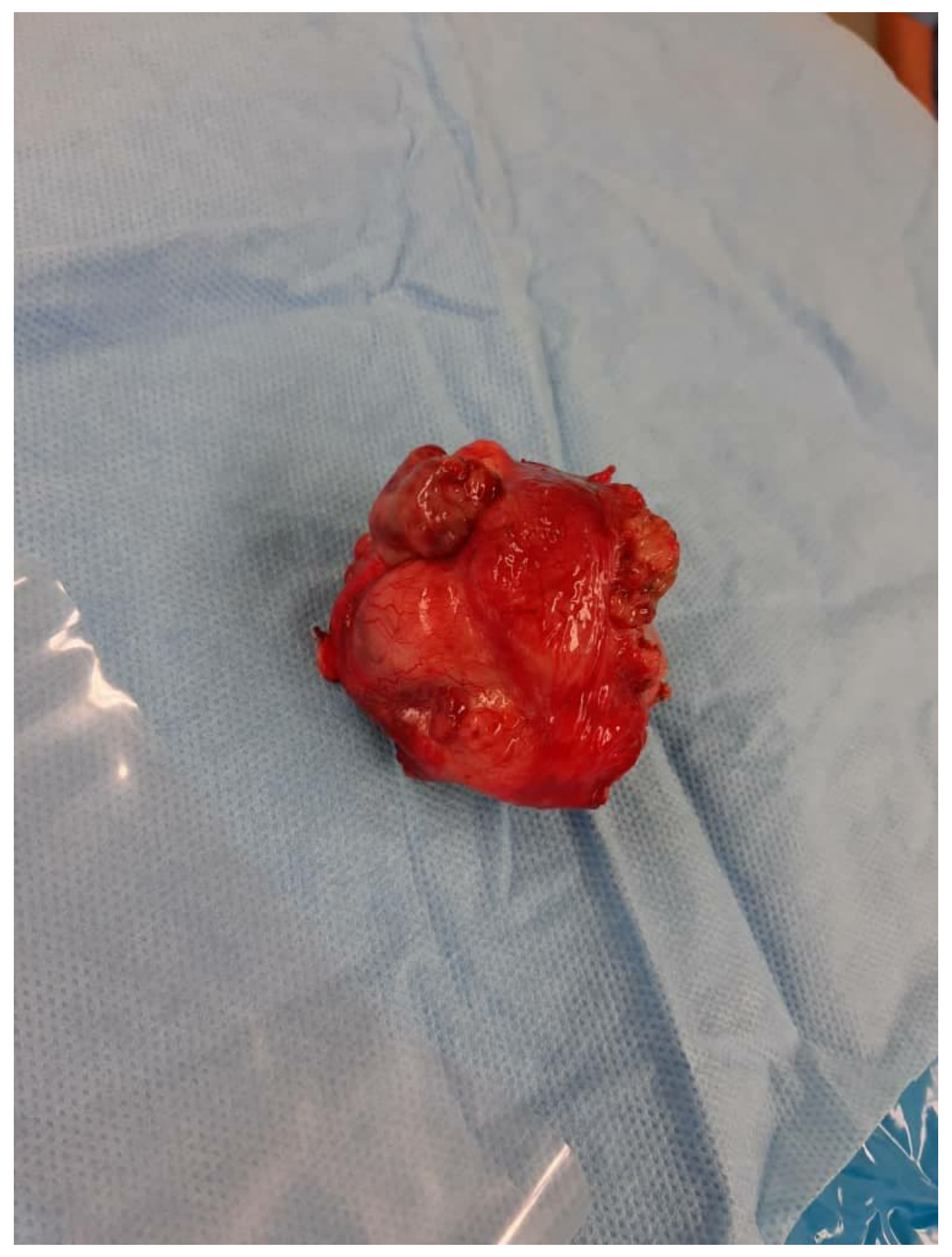

